\title{
Individual behavioral and neuroendocrine changes are correlated with blood-pressure elevations during conflict alone and combined with high-salt diet in baboons
}

\author{
JAYLAN S. TURKKAN \\ The Johns Hopkins University School of Medicine, Baltimore, Maryland
}

\begin{abstract}
Blood pressure, behavioral, and neuroendocrine measures were obtained from adult male baboons during consecutive experimental phases consisting of daily food reinforcement sessions (baseline, 3 weeks), daily food/shock conflict sessions (2 weeks), and enhanced dietary salt added to the daily conflict protocol (3 weeks). Within-session systolic blood pressure (SBP) increased by an average of $5 \% \pm 1 \%$ above baseline during conflict and was accompanied by increases in plasma renin activity, urinary free cortisol, urinary catecholamines, water intake and body weight, and by decreases in urinary sodium excretion in most animals. Behavioral performances of escape from aversive discriminative stimuli and pausing during danger portions of conflict trials increased, while earned food reinforcers decreased. A strong correspondence was found between the degree of individual BP increase during conflict stress and the degree of change in individual stress indexes. Daily dietary salt further elevated SBP by $10 \% \pm 3 \%$ above baseline. Those animals that displayed the greatest changes in physiological and behavioral stress indexes during conflict alone displayed the greatest SBP response during the added high-salt diet. Individuals who are stress-sensitive across a variety of behavioral and physiological systems may be more susceptible to other hypertensinogenic variables.
\end{abstract}

Animals and humans under stress exhibit perturbations in a number of behavioral and physiological systems (Krystal, 1990; Mason, Giller, Kosten, \& Yehuda, 1990; Turkkan, Brady, \& Harris, 1982). Although a single definitive stress index has eluded us, there has been a growing recognition that the assessment of a constellation of measures may be the best strategy for assessing stress susceptibility and mechanisms of stress outcomes (Ottenweller, Natelson, Pitman, \& Drastal, 1990) since individuals may exhibit stress sensitivity in some systems but not in others.

Neuroendocrine secretion in both the sympathoadrenomedullary (SAM) and hypothalamic pituitary adrenocortical (HPA) axes have been hallmark indexes of stress (Goldstein, 1987; Mason et al., 1990) and have been implicated in the pathogenesis of hypertension because of their vasoconstrictive and antinatriuretic actions. This study exposed laboratory baboons to experimental sessions of food/shock conflict, measured changes in a number of behavioral, neuroendocrine, and other physiological mea-

This research was supported by U.S. Public Health Service Grant HL40138, National Institutes of Health. The author thanks David S. Goldstein for assays of catecholamines and also thanks M. K. Story, T. Hux, L. Daley, G. Brinkley, and R. Wurster for technical support. Address correspondence to Jaylan S. Turkkan, Division of Behavioral Biology, Department of Psychiatry and Behavioral Sciences, Johns Hopkins University School of Medicine, 617 Traylor Bldg., 720 Rutland Ave., Baltimore, MD 21205. sures that have "face validity" for stress and that are also mechanistically important in hypertension, and examined across animals the covariation between these measures and stress-induced blood-pressure change.

Studies employing only physiological measures as stress indicators may lack validation that stress was in fact experienced by the experimental subject since autonomic and specifically sympathoadrenergic arousal may be elicited by a broad range of psychological states. One method of providing such validation was employed in the current study by differentially pairing distinct stimuli with aversive versus nonaversive periods within daily food/shock conflict sessions and allowing the animal to escape from such stimuli after daily stress sessions were terminated. It was hypothesized that the ability of such danger and safety stimuli to control overt behavior outside of the sessions could be used as an objective "stress report." Other behavioral indexes of stress studied here included the degree of disruption and "conflict" during sessions, expressed as the amount of time that animals pause during time intervals that are visually cued as dangerous, and decreases in the amount of food reinforcers earned during the food/shock conflict sessions. It has been suggested that adjunctive behavior-specifically, schedule-induced polydipsia-may emerge during stress, perhaps because of its stress-reducing properties (e.g., Devenport, 1978), and this possibility was also evaluated here.

Differential responses across subjects exposed to the same stress may be profitably used as a predictor of in- 
dividual responsiveness to other homeostatically intrusive interventions. For example, individuals who display enhanced physiological and behavioral effects of stress may be more reactive to the effects of dietary salt, a variable often implicated in the pathogenesis of hypertension (Freis, 1976). This study thus also examined whether baboons who display exaggerated susceptibility to stress may be predisposed to develop high blood pressure during dietary salt loading in combination with stress.

\section{METHOD}

\section{Animals}

Four adult male baboons (Papio cynocephalus and $P$. anubis) served as subjects because of their similarity to man in terms of circulatory parameters and biochemistry (e.g., Fridman \& Popova, 1988; McCraw \& Sim, 1972). The animals were surgically intact and were approximately 7 years old and weighed between 20 and $26 \mathrm{~kg}$ (Primate Imports, Long Island, NY). In an earlier study, these animals experienced the same levels of sodium diet and food/shock conflict as used here for 8-16 weeks per condition, both alone and combined (Turkkan \& Goldstein, in press). The animals were in a resting condition for 13 weeks prior to the start of the current baseline measurement period. Eight weeks has been suggested to be a required stress-recovery interval for baboons (Steyn, 1975). Daily diet consisted of $300 \mathrm{~g}$ of ration and one piece of fruit. The standard monkey chow (Purina No. 5037) contained $5 \%$ fat, $0.22 \%$ sodium, $0.34 \%$ chlorine, $0.5 \%$ potassium, and $83 \mathrm{ppm}$ cholesterol. The light:dark cycle of the laboratory was 12:12 h (lights on at $0600 \mathrm{~h}$ ).

\section{Equipment}

The animals were individually housed in aluminum cages (LWH $1.2 \times 0.9 \times 1.4 \mathrm{~m}$ ) containing a seating bench and a water spout easily accessible during experimental sessions; tap water for drinking was available ad lib. An intelligence panel attached to each cage contained a response lever (Lindsley) modified for presentation of mild electric shock and a $7.6 \times 12.7 \mathrm{~cm}$ hinged panel (BRS/LVE No. PJS601 ) at eye level that could be transilluminated with a green or red stimulus hue (safety and danger discriminative stimuli, respectively; $\mathbf{S}^{\mathrm{D}}$.

\section{Procedure}

General protocol. Animals experienced a baseline condition in which they pulled on a lever in order to earn food pellets in daily experimental sessions (BASELINE, 3 weeks). This was followed by a period of food/shock conflict stress during ongoing daily food sessions (CONFLICT, 2 weeks). Animals were next administered a daily diet high in salt content while food/shock conflict sessions continued (CONFLICT + SODIUM, 3 weeks). Experimental conditions for subjects SY and IM were staggered by 1 week to limit contemporaneous exposure.

Behavioral sessions. Behavioral sessions occurred in the living cages twice daily, from 0900 to $1100 \mathrm{~h}$ and from 1200 to $1400 \mathrm{~h}$ on weekdays. Sessions ended after $150 \mathrm{~min}$ or 90 trials, whichever occurred first. Each session was divided into two distinct periods: an operant food schedule period (with or without added conflict stress), followed by a 15 -min period during which the food schedule was terminated but presses on the stimulus panel could change the color illuminated on the panel ("escape from $S^{D}$ "). During the food schedule, the animals earned one $1-\mathrm{g}$ food pellet according to a fixed ratio (FR) 100 schedule of lever pulls in the presence of a green $S^{D}$ illuminated on the intelligence panel. During BASELINE sessions, no other contingencies were in effect. During $30 \%$ of trials in each CONFLICT stress session, lever pulls 97-100 of the trial each had a $35 \%$ probability of producing mild electric shock but did not otherwise affect the FR count. Each shock was pre- signaled with a red $S^{D}$ illuminated on the intelligence panel; pulls during the red $S^{D}$ initiated a $1 / 2-s e c$ electric shock through the lever (AC, constant current; 2-3 mA), after which the red $S^{D}$ was briefly extinguished. The green $S^{D}$ was illuminated during all other portions of the trial. If the animal failed to pull the lever, the red $S^{D}$ remained lit, and the food (or food/shock) schedule period timed out with no further consequences. Trials were separated by 2 -sec intervals during which stimulus lights were extinguished and no other contingencies were in effect.

Following the period when reinforcement was available, the red $S^{D}$ was illuminated on the panel. Presses on the panel changed the panel hue to green for $30 \mathrm{sec}$; repeated presses continued the green hue for $30 \mathrm{sec} / \mathrm{press}$. If $20 \mathrm{sec}$ elapsed without a press, the red $S^{D}$ was reilluminated on the panel and remained lit if no further presses occurred. Only during this "escape from $S^{\mathrm{D}}$ " period did panel presses affect panel hue. Presses on the panel produced no other consequences, and no other events including shocks or food were scheduled during this period. Animals were not trained to press the panel. After $15 \mathrm{~min}$, the panel light was extinguished indicating session termination. Animals were fed at $1500 \mathrm{~h}$; food rations were adjusted daily according to the number of pellets earned in order to maintain a constant diet.

Sodium chloride administration. Enhanced salt diet was administered as $5 \%$ hypertonic saline (Abbott Laboratories, North Chicago, IL) in a flavored water vehicle for ingestion; $300 \mathrm{ml}$ saline was combined with powdered orange drink and tap water in constant total volume of $450 \mathrm{ml} /$ day. This yielded a dose of $15 \mathrm{~g}$ $\mathrm{NaCl} /$ day; otherwise, the same volume of flavored water alone was administered. Drink bottles were presented at $0730 \mathrm{~h}$ on weekdays, $0900 \mathrm{~h}$ on weekends. Solutions were consumed within $30 \mathrm{~min}$.

Cardiovascular measurements. Brachial artery auscultatory systolic blood pressure (SBP) and diastolic blood pressure (DBP) were measured in seated awake baboons in the living cage with a standard mercury manometer and inflatable cuff three times a day, prior to the session onset ("presession," $0830 \mathrm{~h}$ ) and at $0915 \mathrm{~h}$ and 1415 h ("within-session"), Monday-Friday. Animals voluntarily extended an arm outside of the cage onto a shelf during measurement (details in Turkkan, 1990). BPs also were measured with a random zero sphygmomanometer (Hawksley Co., Sussex, England) 1 day per week, 3 times a day in order to assess observer bias. Random-zero BPs did not differ statistically from other BP values and were pooled. Heart rate (HR) was measured by palpation of the brachial artery for $60 \mathrm{sec}$ immediately prior to the BP readings.

Urine collection and assays. Urine was collected over $24-h$ intervals $(0700 \mathrm{~h}-0700 \mathrm{~h})$ on Tuesdays and Thursdays in collection pans with wire mesh $(0.8 \times 1.2 \mathrm{~m}, \mathrm{~W} \times \mathrm{L})$ placed under the individual cages. Urine was collected three times a day and refrigerated to limit evaporation. Electrolyte content was determined by flame photometry, and urinary free cortisol (UFC) was determined by radioimmunoassay. Acidified samples of urinary catecholamines (epinephrine [EPI] and norepinephrine [NE]) were stored at $-70^{\circ} \mathrm{C}$ and were assayed by batch alumina extraction, followed by liquid chromatography with electrochemical detection (Eisenhofer et al., 1986).

Blood collection and assays. Femoral arterial blood was collected for analysis of plasma renin activity (PRA) during the final week of each experimental condition on the same day of the week at $0900 \mathrm{~h}$ (behavioral sessions suspended). Each animal was sedated with ketamine $(5 \mathrm{mg} / \mathrm{kg}$ i.m.), then kept in a supine position for $30 \mathrm{~min}$ during halothane anesthesia while effects of ketamine dissipated. Blood was drawn into coded, chilled glass tubes containing EDTA and cold centrifuged immediately. Plasma was frozen until assay within 30 days. PRA was measured by the rate of angiotensin I generation (Smith Kline \& French Laboratories, Van Nuys, CA).

\section{Data Analysis}

Data were averaged over the final 5 days of each experimental condition, which reflects chronic rather than acute changes. Planned comparisons among the three conditions (BASELINE, CONFLICT, 
CONFLICT + SODIUM) were conducted with paired $t$ tests (twotailed) on both presession and within-session levels of cardiovascular (CV) measures. $P$ levels less than .05 were considered significant. Because of the small number of subjects, statistical analyses ( $t$ tests) were planned for the cardiovascular data only, since the present aim was to describe the physiological and behavioral changes that accompanied the degree of (significant) CV changes observed, across individual baboons.

\section{RESULTS}

\section{Conflict Stress}

Physiological effects. Presession SBP increased from an average of $120 \pm 7 \mathrm{~mm} \mathrm{Hg}$ during BASELINE to $123 \pm$ $7 \mathrm{~mm} \mathrm{Hg}$ during the final (2nd) week of CONFLICT (paired $t$, two-tailed, $p>.10$ ), representing an average increase above baseline of $3 \% \pm 2 \%$. Mean within-session SBP significantly increased from $120 \pm 7 \mathrm{~mm} \mathrm{Hg}$ during BASELINE to $126 \pm 9 \mathrm{~mm} \mathrm{Hg}$ during the final week of CONFLICT $[t(3)=3.25, p<.05]$, representing an average increase above baseline of $5 \% \pm 1 \%$. Figure 1 illustrates the percentage of changes in SBP for individual animals during CONFLICT (DBP and HR were not significantly changed from baseline levels).

Individual animals displayed neuroendocrine effects of conflict stress that generally covaried with their degree of SBP change from baseline (Figure 1). PRA increased from an average of $7.83 \pm 2.5 \mathrm{ng} / \mathrm{ml} / \mathrm{h}$ during BASELINE to an average of $9.90 \pm 1.7 \mathrm{ng} / \mathrm{ml} / \mathrm{h}$ during CONFLICT; 3 out of 4 animals displayed increases in PRA. Baboons IM and SL, who displayed greater elevations in presession and within-session SBP during CONFLICT, also displayed greater elevations in PRA; note the better covariation of PRA with presession SBP than with within-session SBP across subjects.

Three out of 4 animals displayed increases in urinary catecholamines during CONFLICT, with generally larger percentage increases evident in urinary epinephrine (UEPI) compared with urinary norepinephrine (UNE). UEPI increased from $9.71 \pm 0.8 \mu \mathrm{g} /$ day during BASELINE to $12.43 \pm 1.9 \mu \mathrm{g} / \mathrm{day}$, while UNE (Figure 1) increased from $8.54 \pm 1.3 \mu \mathrm{g} /$ day during BASELINE to $9.73 \pm$ $1.8 \mu \mathrm{g} /$ day. Among individual subjects, Baboons IM and SL, who had displayed the greatest increases in SBP, also displayed the greatest elevations in levels of urinary catecholamines during CONFLICT.

UFC increased from an average of $161.0 \pm 13.1 \mu \mathrm{g} / 24 \mathrm{~h}$ to an average of $217.67 \pm 49.7 \mu \mathrm{g} / 24 \mathrm{~h}$ during CONFLICT; 3 out of 4 animals displayed increases in UFC (Figure 1). The greatest increases in UFC again were obtained in those animals that showed larger increases in SBP (Baboons IM and SL). Note, however, that the relationship between change in SBP and UFC was not exact in that Baboon IM displayed an intermediate change in SBP but displayed the greatest change in UFC.

Sodium retention (decreases in $\mathrm{U}_{\mathrm{NA}} \mathrm{V}$ ) occurred in 3 out of 4 animals during CONFLICT (Figure 1). Average
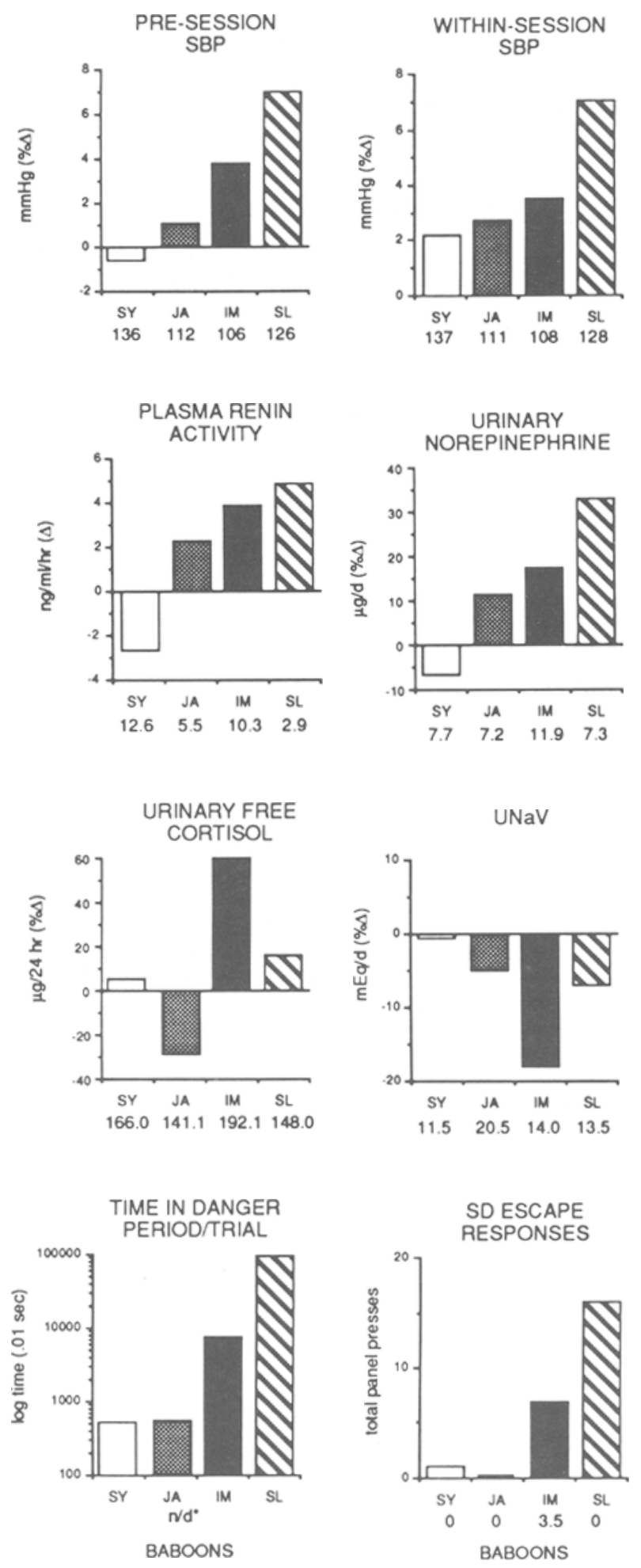

Figure 1. Average individual-subject physiological and behavioral dependent measures during the 3rd week of CONFLICT. Average levels from the final 5 days of BASELINE are shown below each data column. $U_{N A} V=$ sodium concentration in 24 h urine samples. *n/d $=$ there were no danger periods during BASELINE trials. 

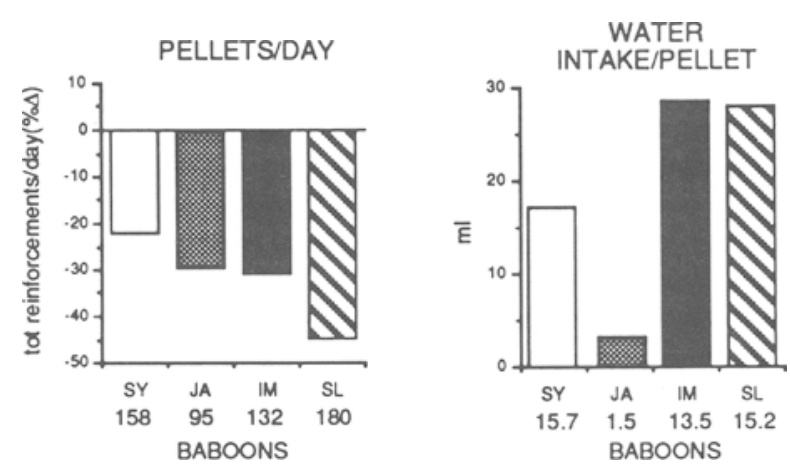

Figure 2. Average individual-subject pellets/day (left panel) and water intake/pellet (right panel) during the 3rd week of CONFLICT. Average levels from the final 5 days of BASELINE are shown below each data column.

$\mathrm{U}_{\mathrm{NA}} \mathrm{V}$ was $14.85 \pm 2.3 \mathrm{mEq} /$ day during BASELINE and decreased to $11.83 \pm 2.2 \mathrm{mEq} /$ day. Baboons IM and SL excreted less sodium compared with Baboons JA and SY. Similar to the pattern across subjects of UFC increases during CONFLICT, Baboon IM again displayed the greatest change in $\mathrm{U}_{\mathrm{NA}} \mathrm{V}$.

Behavioral effects. Changes in behavioral measures across subjects generally covaried with the degree of individual SBP increase during CONFLICT (Figure 1, bottom row). The duration of time in the danger segments of shock trials averaged $474 \pm 486 \mathrm{sec} /$ trial and varied greatly across animals. Baboons who displayed larger SBP increases during CONFLICT took one and two orders of magnitude longer to complete the danger portions of shock trials ( 150 and 1,734 sec, Baboons IM and SL, respectively), as compared with the animals who displayed smaller increases in SBP who took approximately $6 \mathrm{sec}$ to complete danger portions of trials (Baboons JA and SY).

Postsession $S^{D}$ escape responses averaged $6.0 \pm 4.2$ panel presses during CONFLICT (Figure 1). The number of $S^{D}$ escape responses was greater in the animals that showed larger increases in SBP (7 and 16 presses for $\mathrm{Ba}$ boons IM and SL, respectively), as compared with nearly zero presses for the animals showing modest changes in SBP (Baboons JA and SY). Figure 2 shows daily SBP and $S^{D}$ escape responses for Baboon $S L$, who displayed the greatest increase in SBP and the greatest number of escape responses during CONFLICT. SBP tended downward during the final days of BASELINE, and the panel was virtually never pressed during this period. After 2 days of CONFLICT, both SBP and $S^{D}$ escapes increased and tended to covary thereafter; these two measures were significantly correlated across the 20 days shown (Pearson $r$ $=0.465, p<.05$ ).

Decreases in pellets earned per day during CONFLICT also covaried with the degree of SBP increases across animals (Figure 3). Furthemore, Baboons IM and SL, who displayed greater increases in SBP, also displayed the largest amounts of water intake per pellet during CONFLICT (across all animals, mean water intake per pellet during CONFLICT $=19.2 \pm 6.9 \mathrm{ml}$; Figure 3).

Finally, the relationship between BASELINE level and change during CONFLICT was assessed by computing Pearson $r$ and regression coefficients for all physiological and behavioral dependent measures (BASELINE level $\times \% \Delta$ during CONFLICT). BASELINE levels of physiological or behavioral measures did not determine the magnitude of change in these measures during CONFLICT (all $r$ and $\beta p$ values $>.06$; range of $p$ values $=.06-.97$ ).

$B P$ response to dietary salt loading added to conflict stress. Adding daily enhanced dietary salt to ongoing food/shock conflict stress further increased within-session SBP in all animals (Figure 4) by an average of $10 \% \pm 3 \%$ above BASELINE (mean $=132 \pm 7 \mathrm{~mm} \mathrm{Hg} ; p<.05$ for both BASELINE $[t(3)=3.88]$ and CONFLICT alone $[t(3)=3.50]$ comparisons). Peak increases in SBP occurred in the second week of CONFLICT + SODIUM, and averaged $12 \% \pm 2 \%$ above BASELINE levels. Presession

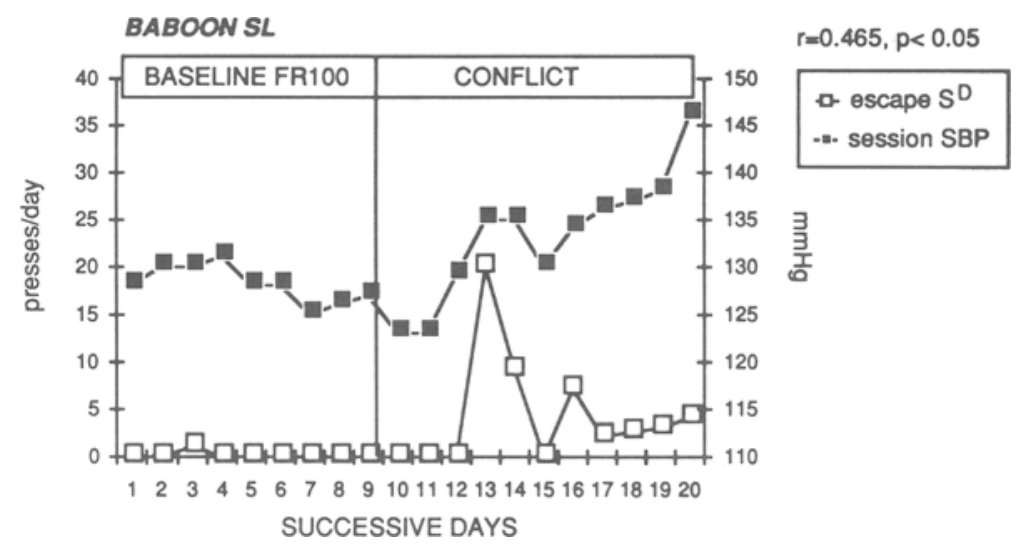

Figure 3. Escape from $S^{D}$ responses (open squares, left ordinate) and within-session SBP (filled squares, right ordinate) during 20 successive experimental session-days for Baboon SL. CONFLICT sessions were initiated at the vertical line. Note the covariation between panel pressing and SBP trends. 


\section{WITHIN-SESSION SBP}

\begin{tabular}{|l|l|}
\hline BASE & CONFLICT \\
\hline & HIGH SODIUM DIET \\
\hline
\end{tabular}

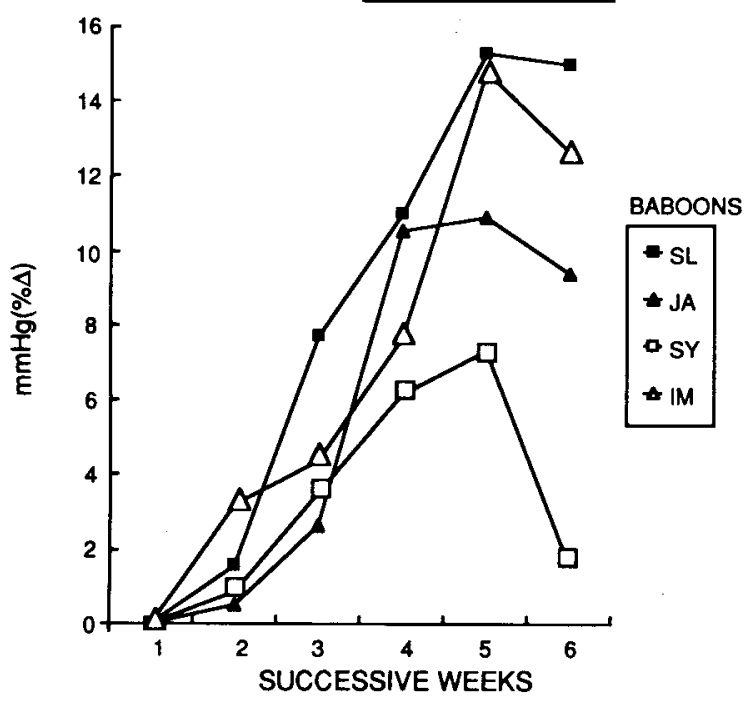

Figure 4. Within-session SBP percentage change from BASELINE as a function of successive weeks. Data from individual baboons are shown in individual functions. Transition points for successive experimental conditions are indicated. Each data point is an average of two daily measurements, averaged over 5 days. BASE = BASELINE.

SBP during CONFLICT + SODIUM was not significantly changed. Also, neither presession nor within-session DBP or HR was significantly changed during CONFLICT + SODIUM.

The degree of SBP increase during CONFLICT + SODIUM was related across animals to the degree of SBP increase above BASELINE during the immediately preceding CONFLICT alone period (Figure 4). In the 2nd and 3rd weeks of CONFLICT + SODIUM, Baboons IM and SL again displayed the largest increases in within-session SBP (13\% and $15 \%$ above BASELINE; $8 \%$ and $11 \%$ above CONFLICT levels) compared with Baboons JA and SY (9\% and $2 \%$ above BASELINE; $6 \%$ and $2 \%$ above CONFLICT levels, respectively).

\section{DISCUSSION}

This study found increases in SBP after intact, genetically unselected and untethered baboons were exposed to daily food/shock conflict stress sessions for 2 consecutive weeks. Although average SBP increases during conflict stress alone were modest, they were accompanied by a variety of changes both in physiological and behavioral systems including increases in UFC, PRA, urinary catecholamines, sodium retention, adjunctive water intake, escape from aversive discriminative stimuli, and pausing during danger portions of shock trials, in conjunction with overall decreases in earned food pellets. The degree of change in these stress-index variables during
CONFLICT generally covaried with the individual degree of SBP increase across animals and was not predicted by BASELINE levels of the measures. Adding enhanced dietary salt to the chronic food/shock conflict experience further elevated SBP and appeared to exert the greatest BP elevations in those animals who could be identified as "stress-sensitive" by a broad range of behavioral and neuroendocrine measures.

Although many studies have examined the effects of acutely administered stress, comparatively few have examined the physiological accompaniments of chronic stress. Acute, but not always chronically administered aversive events typically have been associated with SAM and HPA axis activation in many species, including humans and nonhuman primates (Blair, Feigl, \& Smith, 1976; Levine, Gordon, Peterson, \& Rose, 1970; Panel Report, 1982). The chronic food versus shock stress used in the present study was chosen to induce a conflict that may be viewed as analogous to situations humans commonly face and that has been found to be ulcerogenic and hypertensinogenic in related animal studies (Ader, 1971; Lawler, Barker, Hubbard, \& Allen, 1980). Because the shock intensity was low, animals did not stop pulling the lever altogether, which allowed the conflict stress to be maintained over weeks.

This type of chronic stress experience may be markedly different from stressors that have been typically studied in animals, most notably immobilization stress and inescapable shock. Chronic inescapable stress has resulted in low levels of glucocorticoids (see Mason et al., 1990), in contrast to increased cortisol secretion in those animals who had BP increases in the present study. These differences in physiological responses may have resulted from the "escapable" aspect of the conflict experience (cf. Weiss, Stone, \& Harrell, 1970) in that animals could choose not to pull the electrified lever since it was cued by a discriminative stimulus.

PRA was increased in most baboons during conflict, and the largest PRA elevations occurred in animals showing the greatest sodium retention during conflict. Persistent increases in PRA stimulate the adrenal synthesis of aldosterone, which in turn promotes the reabsorption of sodium by the distal tubules of the kidney and thus the retention of water by the vasculature. The magnitude of change in PRA was small and may have resulted from measurement of PRA concentrations in blood drawn during sedation 1 day following the experimental sessions.

Behavioral perturbations accompanied the physiological changes during conflict stress: The animals earned less food during experimental sessions, they spent more time completing trials because they paused during danger portions of trials, and 2 animals worked to turn off stimuli associated with conflict stress. When initially neutral stimuli are paired with aversive/stressful events, they may come to exert effects on the organism when physical stressors are not present, thereby potentially multiplying the number of stressful stimuli in the organism's environment (e.g., Overmier \& Murison, 1989). The $S^{D}$ escape pro- 
cedure employed here allowed the animals to change a visual stimulus in their living cage from a danger to a safety hue, thus providing not only an ongoing picture of associations forming between visual cues and stress, but more importantly providing a continuous real-time report by the animal of the aversiveness of the preceding stress session. Animals that showed greater physiological and other behavioral effects of stress also worked more to remove stress-associated stimuli from their visual environment. Together with physiological indexes of stress, the aversive stimulus escape method seems promising as a separate but converging objective index of stress that is not muscular effort-intensive. The validity of the $S^{D}$ escape procedure is supported by studies showing that animals will work to turn off stimuli paired with aversive events such as extinction (Daly \& McCroskery, 1973), drug withdrawal states (Goldberg, 1976), and shock delivery (Rilling, Askew, Ahlskog, \& Kramer, 1969).

We have previously found that hyperdipsia emerges reliably in laboratory baboons during sessions containing both electric shock and food reinforcement delivered intermittently (Turkkan \& Harris, 1984). The hyperdipsia observed during the present food/shock conflict period satisfied previously published criteria of adjunctive polydipsia and reached $>25 \mathrm{ml} / \mathrm{g}$ of food in animals who showed greater changes in stress indexes during conflict. Polydipsia has emerged during aversive experimental sessions (e.g., Dantzer et al., 1988), has been suggested to be critically associated with HPA axis activity (Devenport, 1978), and has been suggested to have stress-reducing properties (Tazi, Dantzer, \& Le Moal, 1988).

Blood pressure of all animals increased further when dietary salt was added to the conflict protocol and by double the degree of BP increase found previously in baboons administered chronic salt loading alone (Turkkan \& Goldstein, 1987) or chronic food/shock conflict stress alone (Turkkan \& Goldstein, in press). Individual differences in BP sensitivity to dietary salt loading were, however, apparent. There has been a great deal of speculation as to the determinants of BP salt-sensitivity in humans (e.g., Luft, Weinberger, Grim, Fineberg, \& Miller, 1983). The results of the current study suggest that one of the predictors of BP salt-sensitivity may be a prior or ongoing state of exaggerated response to stress. After high salt was added to the diet, the largest SBP increases were obtained in those animals undergoing behavioral and physiological manifestations of stress.

Suggestive findings that the combination of sodium and stress/arousal variables may be synergistic for acute blood-pressure elevations is provided by evidence that sodium chloride may sensitize vascular response to sympathetic stimulation (Rankin, Luft, Henry, Gibbs, \& Weinberger, 1981; Sakamaki et al., 1984). Increased sympathetic stimulation during the present conflict stress was indexed by increases in urinary catecholamines, reflecting increases in circulating catecholamines. This substrate of enhanced adrenomedullary and sympathoneural activity during ongoing stress may have enhanced the vascular response to dietary salt loading in baboons. At least one disconfirmatory report has appeared, however, showing that genetically borderline hypertensive rats experiencing stress failed to increase BP further when administered a diet high in salt content (Sanders, Cox, \& Lawler, 1988). As noted above, the stress used in the present study was escapable (or indefinitely delayable), as opposed to the inescapable shock used in Sanders et al. (1988) and in the majority of stress studies, which may have blunted potential BP increases.

There is accumulating evidence that exaggerated response to stress may be an enduring and perhaps heritable characteristic of individuals (Roske, Umatov, Pevcova, Rathsack, \& Oehme, 1988; Taylor, Weyers, Harris, \& Vogel, 1989). The current data suggest that exaggerated response to chronic stress may create an enduring biobehavioral substrate that predisposes individuals to the hypertensinogenic effects of other variables.

\section{REFERENCES}

ADER, R. (1971). Experimental investigations: Experimentally induced gastric lesions. Advances in Psychosomatic Medicine, 6, 1-39.

Blair, M. L., Feigl, E. O., \& SMITh, O. A. (1976). Elevation of plasma renin activity during avoidance performance in baboons. American Journal of Physiology, 231, 772-776.

DAlY, H. B., MCCROSKERY, J. H. (1973). Acquisition of a bar-press response to escape frustrative non-reward and reduced reward. Journal of Experimental Psychology, 98, 109-112.

Dantzer, R., Terlouw, C., Tazi, A., Koolhaas, J. M. et al. (1988). The propensity for schedule-induced polydipsia is related to differences in conditioned avoidance behavior and in defense reactions in a defeat test. Physiology \& Behavior, 43, 269-273.

DEVENPORT, L. D. (1978). Schedule-induced polydipsia in rats: Adrenocortical and hippocampal modulation. Journal of Comparative \& Physiological Psychology, 92, 651-660.

Eisenhofer, G., Goldstein, D. S., Stull, R., Keiser, H. R., SunDERLAND, T., MURPHY, D. L., \& KoPIN, I. J. (1986). Simuitaneous liquid chromatographic determination of 3,4-dihydroxyphenylglycol, catecholamines, and 3,4-dihydroxyphenylalanine in plasma and their responses to inhibition of monoamine oxidase. Clinical Chemistry, 32, 2030-2033.

FrEIS, E. D. (1976). Salt, volume and the prevention of hypertension. Circulation, 53, 589-595.

Fridman, E. P., \& Popova, V. N. (1988). Species of the genus Papio (Cercopithecidae, Primates) as subjects of biomedical research: I. Biological basis of experiments on baboons. Journal of Medical Primatology, 17, 291-307.

GoldberG, S. R. (1976). Stimuli associated with drug injections as events that control behavior. Pharmacological Reviews, 27, 325-340.

GoldsteIN, D. S. (1987). Stress-induced activation of the sympathetic nervous system. In A. Grossman (Ed.), Neuroendocrinology of stress (pp. 253-278). London: Bailliere-Tindall.

Krystal, J. H. (1990). Animal models for posttraumatic stress disorder. In E. L. Giller (Ed.), Biological assessment and treatment of posttraumatic stress disorder (pp. 1-26). Washington, DC: American Psychiatric Press.

Lawler, J. E., Barker, G. F., Hubbard, J. W., \& Allen, M. T. (1980). The effects of conflict on tonic levels of blood pressure in the genetically borderline hypertensive rat. Psychophysiology, 17, 363-370.

Levine, M. D., Gordon, T. P., Peterson, R. H., \& Rose, R. M. (1970). Urinary 17-OHCS response of high- and low-aggressive rhesus monkeys to shock avoidance. Physiology \& Behavior, 5, 919-924. 
Luft, F. C., Weinberger, M. H., Grim, C. E., Fineberg, N. S., \& MilleR, J. Z. (1983). Sodium sensitivity in normotensive human subjects. Annals of Internal Medicine, 98, 758-762.

Mason, J. W., Giller, E. L., Kosten, T. R., Yehuda, R. (1990). Psychoendocrine approaches to the diagnosis and pathogenesis of posttraumatic stress disorder. In E. L. Giller (Ed.), Biological assessment and treatment of posttraumatic stress disorder (pp. 65-86). Washington, DC: American Psychiatric Press.

McCraw, A. P., \& Sim, A. K. (1972). Clinical biochemistry of the baboon. Journal of Comparative Pathology, 82, 193.

Ottenweller, J. E., Natelson, B. H., Pitman, D. L., \& Drastal, S. (1990). Adrenocortical and behavioral responses to repeated stressors: Toward an animal model of chronic stress and stress-related mental illness. Biological Psychiatry, 26, 829-841.

Overmier, J. B., \& Murison, R. (1989). Poststress effects of danger and safety signals on gastric ulceration in rats. Behavioral Neuroscience, 103, 1296-1301.

Panel Report on Biological Substrates of Stress (1982). In G. R. Elliott \& C. Eisdorfer (Eds.), Stress and human health (pp. 189-254). New York: Springer.

Rankin, L. I., LuFt, F. C., Henry, D. P., Gibes, P. S., \& WeinBERGER, M. H. (1981). Sodium intake alters the effects of norepinephrine on blood pressure. Hypertension, 3, 650-656.

Rilung, M., Askew, H. R., AHLSKOG, J. E., \& KRAMER, T. J. (1969). Aversive properties of the negative stimulus in a successive discrimination. Journal of the Experimental Analysis of Behavior, 12, 917-932.

Roske, I., Umatov, E. A., Pevcova, E. I., Rathsack, R., \& Oehme, P. (1988). Influence of adrenal demedullation on stress-induced alterations within the general adaptation behavior of August rats. Pharmazie, 43, 339-343.

Sakamaki, T., Johnson, J. A., Zeigler, D. W., Koivunen, D. G., Siripaisarnpipat, S., Fowler, W. L., JR., \& Payne, C. G. (1984). Pressor hyperresponsiveness in saline-infused rabbits. Hypertension, 6, 503-510.
Sanders, B. J., Cox, R. H., \& LAWLer, J. E. (1988). Cardiovascular and renal responses to stress in borderline hypertensive rat. American Journal of Physiology, 255, R431-R438.

STEYN, D. G. (1975). The effects of captivity stress on the blood chemical values of the chacma baboon (Papio ursinus). Laboratory Animals, 9, 111-120.

Taylor, J., Weyers, P., Harris, N., \& Vogel, W. H. (1989). The plasma catecholamine stress response is characteristic for a given animal over a one-year period. Physiology \& Behavior, 46, 853-856.

Tazi, A., Dantzer, R., Le Moal, M. (1988). Schedule-induced polydipsia experience decreases locomotor response to amphetamine. Brain Research, 445, 211-215.

TURKKaN, J. S. (1990). New methodology for measuring blood pressure in awake baboons with use of behavioral training techniques. Journal of Medical Primatology, 19, 455-466.

Turkkan, J. S., Brady, J. V., \& Harris, A. H. (1982). Animal studies of stressful interactions: A behavioral-physiological overview. In L. Goldberger \& S. Breznitz (Eds.), Handbook of stress: Theoretical and clinical aspects (pp. 153-182). New York: Free Press.

TurkKan, J. S., \& Goldstein, D. S. (1987). Production and reversal of DOCA/salt hypertension in baboons. Clinical \& Experimental Hypertension (A), 9, 125-140.

TuRkKan, J. S., \&oldsteIN, D. S. (in press). Chronic effects of high salt intake and conflict stress on blood pressure in primates: A progress report. Integrative Physiological \& Behavioral Science.

TurkKan, J. S., \& HaRRIS, A. H. (1984). Hyperdipsia in the baboon during operant conditioning of blood pressure elevations. Behavioral Neuroscience, 98, 631-639.

Weiss, J. M., Stone, E. A., \& HARrell, N. (1970). Coping behavior and brain norepinephrine levels in rats. Journal of Comparative \& Physiological Psychology, 72, 153-160.

(Manuscript received December 3, 1990; revision accepted for publication February 18, 1991.)

\title{
Notices and Announcements
}

\author{
32nd Annual Meeting of the Psychonomic Society \\ San Francisco, California \\ November 22-24, 1991
}

The 32nd Annual Meeting of the Psychonomic Society will be held in San Francisco, November 22-24, 1991. The meetings will begin Friday morning and continue until Sunday at noon. The headquarters hotel will be the Hyatt Regency.

The call for papers was mailed to members and associates in April. The deadline for submissions is June 21.

The program and hotel reservation cards will be mailed to members and associates in September. A copy of the program will be published in the November issue of the Bulletin of the Psychonomic Society.

For further information, please contact the secretary-treasurer of the Society: Cynthia H. Null, P.O. Box 7104, San Jose, California 95150-7104 (telephone: 415-604-1260). 